\title{
EFFECTS OF MYOCARDIAL ISCHEMIA ON THE RELEASE OF CARDIAC TROPONIN I IN ISOLATED RAT HEARTS
}

Sidney Chocron, $\mathrm{MD}^{\mathrm{a}}$

Kifah Alwan, $\mathrm{MD}^{\mathrm{a}}$

Gerard Toubin, $\mathrm{MD}^{\mathrm{b}}$

Bernadette Kantelip, MD $^{\mathrm{c}}$

François Clement, $\mathrm{MD}^{\mathrm{a}}$

Jean-Pierre Kantelip, MD ${ }^{b}$

Joseph-Philippe Etievent, $\mathrm{MD}^{\mathrm{a}}$
Background: The twofold aim of this experimental study was (1) to verify the correlation between the duration of ischemia and concentration of cardiac troponin I and (2) to compare the release of cardiac troponin I with histologic findings. Methods: Experiments were done on 18 rat hearts, which were perfused according to the Langendorff method, immediately after excision in group I (control group) and after immersion for 3 hours (group II) and 6 hours (group III) in St. Thomas' Hospital solution at $4^{\circ}$ C. During reperfusion, the release of cardiac troponin $\mathrm{I}$, creatine kinase isoenzyme $\mathrm{MB}$, and lactate dehydrogenase, the recovery of left ventricular pressure, and heart rates were compared among the three groups. After the experiment, three samples of myocardium (left ventricle, right ventricle, and septum) were taken for histologic examination. Results: Cardiac troponin I concentration was significantly higher in group III than in groups I and II and in group II compared with group I. Cardiac troponin I concentration increased as the ischemic period increased. The relation between cardiac troponin I release and ischemic duration tended to be linear. Creatine kinase MB and lactate dehydrogenase concentrations did not differ from one group to the other. Left ventricular pressure was not significantly different among the groups. In the control group, no heart had more than $10 \%$ of the myocytes affected. One of six hearts in group II and three of six in group III had more than $10 \%$ of myocytes affected. Conclusion: This experimental study showed (1) that cardiac troponin $I$ is an early marker of ischemic injury and (2) that cardiac troponin I concentration increases as the ischemic period increases. Early cardiac troponin I release appears to correlate with the extent of ischemic injury in rats undergoing buffer perfusion. (J Thorac Cardiovasc Surg 1996;112:508-13)

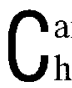
ardiac troponin I has already been shown to be a highly specific marker of acute myocardial infarction ${ }^{1-4}$ and of reperfusion after thrombolytic therapy. ${ }^{1} \mathrm{We}^{5}$ previously reported cardiac troponin I to be a marker of perioperative myocardial ischemia. In a group of 20 patients undergoing aortic valve replacement for pure aortic stenosis with normal coronary arteries, a positive significant cor-

From the Departments of Thoracic and Cardiovascular Surgery, ${ }^{2}$ Pharmacology, ${ }^{\mathrm{b}}$ and Pathology, ${ }^{\mathrm{c}}$ Saint-Jacques Hospital, Besançon, France.

Received for publication Sept. 11, 1995; revisions requested Oct. 25, 1995; revisions received Nov. 28, 1995; accepted for publication Jan. 16, 1996.

Address for reprints: Sidney Chocron, MD, Department of Thoracic and Cardiovascular Surgery, Saint-Jacques Hospital, 25030, Besançon, France.

Copyright @e 1996 by Mosby-Year Book, Inc.

$0022-5223 / 96 \$ 5.00+0 \quad \mathbf{1 2 / 1 / 7 1 9 1 8}$ relation existed between aortic crossclamp time and cardiac troponin I concentration at hour 6. This correlation was modest $(r=0.6)$. The twofold aim of this experimental study was (1) to verify the correlation between the duration of ischemia and concentration of cardiac troponin I and (2) to compare cardiac troponin I release with histologic findings.

\section{Methods}

Experimental protocol. Eighteen male Wistar rats, weighing 400 to $450 \mathrm{gm}$, were anesthetized intraperitoneally with urethane $1 \mathrm{gm} / \mathrm{kg}$. After intravenous administration of sodium heparin (500 IU), the aorta was crossclamped and a St. Thomas' Hospital cardioplegic solution at $4^{\circ} \mathrm{C}$ was injected into the aortic root until ischemic arrest occurred. The heart was then excised.

Hearts from the 18 rats were divided into three experimental groups. In group I, the hearts were suspended immediately on the perfusion apparatus by attaching the aorta to the perfusion cannula and were perfused accord- 
ing to the Langendorff method with an oxygenated KrebsHenseleit bicarbonate buffer containing (in millimoles per liter) $\mathrm{NaCl} 118.0, \mathrm{KCl} 4.7, \mathrm{CaCl}_{2} 1.25, \mathrm{MgSO}_{4}$ 1.2, $\mathrm{KH}_{2} \mathrm{PO}_{4} 1.2, \mathrm{NaHCO}_{3} 25.0$, and glucose 11.0. The solution was equilibrated at $37^{\circ} \mathrm{C}$ with $95 \%$ oxygen and $5 \%$ carbon dioxide. The hydrostatic perfusion pressure was set at $120 \mathrm{~cm} \mathrm{H}_{2} \mathrm{O}$. A water-filled latex balloon connected to a tube was inserted and positioned in the left ventricle through the left atrium for measurement of the left ventricular pressure (LVP). End-diastolic pressure was set at $10 \mathrm{~cm} \mathrm{H}_{2} \mathrm{O}$. If necessary, the heart was defibrillated. In group II, this procedure was performed after 3 hours of immersion in a St. Thomas' Hospital solution at $4^{\circ} \mathrm{C}$. In group III, the hearts were immersed in St. Thomas' Hospital solution for 6 hours.

The hearts were allowed to stabilize for 30 minutes. Effluent samples were collected at baseline and 20, 40,60, 90 , and 120 minutes after stabilization. During reperfusion, the release of cardiac troponin $\mathrm{I}$, creatine kinase isoenzyme $\mathrm{MB}$ (CK-MB), and lactate dehydrogenase (LD), the recovery of LVP, and heart rates were compared among the three groups at each collection.

Histologic analysis. After the experiment, each heart was cut open and segments of right and left ventricles and septum were removed immediately. All segments were fixed for morphologic study in cacodylate-buffered $2 \%$ glutaraldehyde, postfixed in $1 \%$ osmium tetroxide phosphate buffer, dehydrated, and then embedded in Epon fixative. Semithin sections ( $1 \mu \mathrm{m}$ thick) were prepared and stained with alkaline toluidine blue for light microscopic study. Three samples of each segment removed were studied for each heart. The affected myocytes, isolated or grouped, appeared to be swollen with or without contraction bands.

Two histologic groups were defined. Group A included hearts in which fewer than $10 \%$ of the myocytes were affected $(n=14)$. Group B included hearts having at least one sample in which more than $10 \%$ of the myocytes were affected $(n=4)$.

Assay of cardiac troponin I, CK-MB, and LD. Cardiac troponin I concentrations were measured by an enzymelinked immunoenzymetric assay developed by ERIA Diagnostics Pasteur (Marne-la-Coquette, France). The assay is based on a sandwich technique that allows a quantitative determination of the cardiac troponin $I$ in serum. The solid phase is a polystyrene tube coated with an anticardiac troponin I monoclonal antibody (MAb 8E1). Revelation is performed with a second anticardiac troponin I monoclonal antibody (MAb 11E12). Each monoclonal antibody is directed at a different peptide sequence of cardiac troponin I. ${ }^{6}$ The colored reaction occurs only if both monoclonal antibodies are bound to the protein. The absorbance was read at $450 \mathrm{~nm}$ on the status spectrophotometer. Results were expressed as micrograms per liter.

The method used for CK-MB assay was based on Rosalki's spectrophotometric procedure. ${ }^{7}$ Serum samples were incubated with a CK-MB reagent containing an antibody specific to the CK-M subunit that completely inhibits the CK-M monomer. The activity of the CK-B, which was not inhibited by the antibody, was determined after a reaction sequence, by measuring the rate of change in absorbance measured at $340 \mathrm{~nm}$.
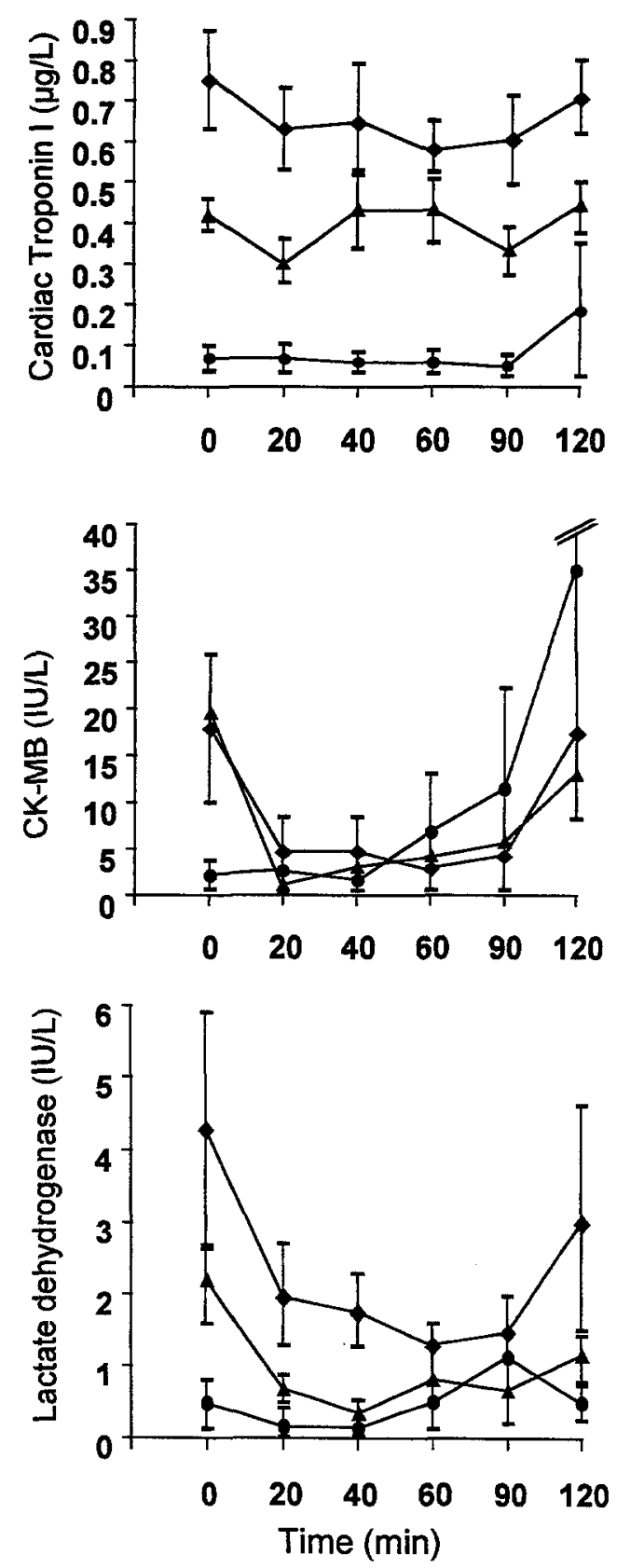

Fig. 1. Time course of cardiac troponin I, CK-MB, and LD release in the control group (group I, black circles), in the group with 3 hours of ischemia (group II, black triangles), and in the group with 6 hours of ischemia (group III, black diamonds). The curves of cardiac troponin I are significantly higher in group III than in groups I and II and in group II compared with group I $(p<0.01)$. During the time course, within each group, the variation of cardiac troponin $I$ is not significant. The curves of CK-MB and LD do not differ $(p>0.20)$ from one group to the other. 

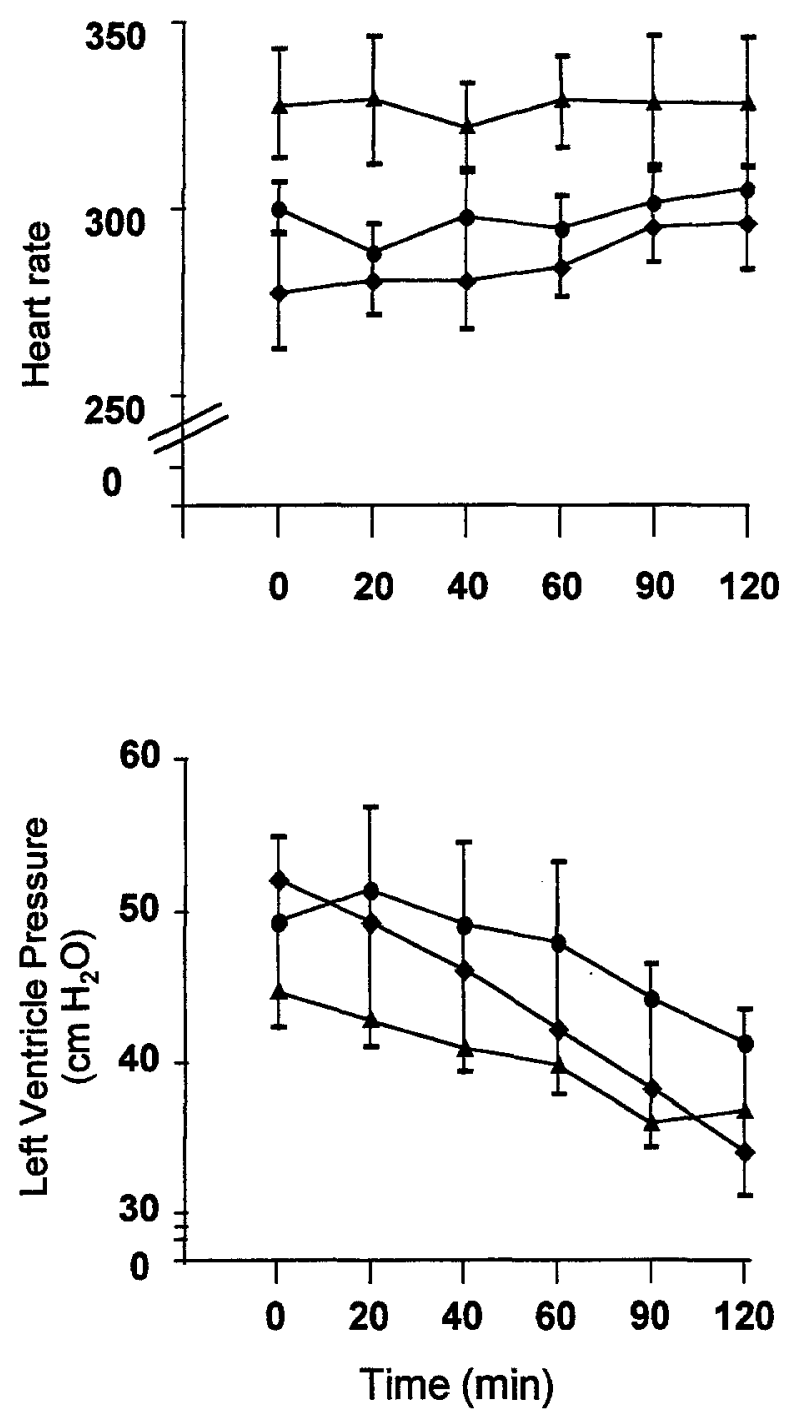

Fig. 2. Time course of left ventricular pressure and heart rate in the control group (group I, black circles), in the group with 3 hours of ischemia (group II, black triangles), and in the group with 6 hours of ischemia (group III, black diamonds). The heart rate curve is significantly higher in group II than in group III. The left ventricular pressure curve does not significantly differ from one group to the other.

The method used for LD assay was based on Wroblewski and LaDue's spectrophotometric procedure. ${ }^{8}$ LD catalyzes the conversion of pyruvate to L-lactate while the reduced form of nicotinamideadenine dinucleotide is oxidized. The rate of oxidation is proportional to LD activity. The activity is monitored by measuring the decrease in absorbance at $340 \mathrm{~nm}$. Results are expressed as international units per liter.

Statistical analysis. Values are expressed as mean \pm standard error of the mean. The averages of cardiac troponin I, CK-MB, LD, LVP, and heart rate among the groups were compared by means of a one-way analysis of variance. When $\mathrm{F}$ values indicated that significant differences were present, we then used Bonferroni's significant difference method, which adjusts for multiple comparisons, to compare samples for statistical significance.

A test for linear trend was carried out for cardiac troponin I, CK-MB, and LD concentrations throughout the course of the three ordered periods of ischemia. The closeness of the association between two variables is usually measured by the product-moment correlation coefficient $r$. The use of this statistic might be thought objectionable, particularly when the measurements to be analyzed are qualitative, although ordered. ${ }^{9}$ Conversely, instead of analyzing differences between group means, we may want to look for trends across the means, provided that the levels of the grouping factor are ordered. For example, a significant linear trend would indicate that the means increase or decrease in a linear fashion.

The total amount of cardiac troponin I released during the reperfusion in groups $A$ and $B$ was compared with the Wilcoxon rank sum test. The statistical analysis was performed with BMDP statistical software (BMDP Corp., Los Angeles, Calif.).

\section{Results}

No stunning occurred during early reperfusion. Fig. 1 shows the time course of cardiac troponin I, CK-MB, and LD release in each group. Analysis of variance shows that the curves were significantly different only for cardiac troponin I $(p<0.01)$. The concentration of cardiac troponin I was significantly higher at each collection in group III than in groups I and II and in group II compared with group I.

Within each group, there was a small, nonsignificant variation of cardiac troponin I during the time course. Cardiac troponin I concentration increased as the ischemic period increased. Statistical analysis revealed that the relation between cardiac troponin I release and ischemic duration tended to be linear ( $p$ ranged from 0.00001 to 0.004 depending on the time of collection). CK-MB and LD concentrations were not linked to the duration of ischemia. LVP was not significantly different among groups. Heart rate was significantly higher in group II than in group III (Fig. 2).

Rat weight, heart weight, the amount of St. Thomas' Hospital solution injected into the aortic root, and the number of shocks necessary to defibrillate the hearts were not significantly different from one group to the other (data not shown).

In the control group, no heart had more than $10 \%$ of the myocytes affected. One heart of six in group II and three of six in group III had more than $10 \%$ of the myocytes affected. 
Total release of cardiac troponin I during reperfusion was clearly higher in group B than in group A $(4.85 \pm 1.23 \mu \mathrm{g}$ versus $2.37 \pm 0.50 \mu \mathrm{g})$. This difference was not significant $(p=0.09)$ because of the number of hearts in each group (14 in group $A$ versus 4 in group B).

\section{Discussion}

Validation of Diagnostics Pasteur cardiac troponin $I$ assay in rats. To the best of our knowledge, this is the first report on cardiac troponin I release in an experimental study involving rats and using the Diagnostic Pasteur assay. Thus the use of this particular cardiac troponin I assay in rats has not been validated. Two arguments may show this assay to be valid in rats. First, the amino acid sequences of cardiac troponin $I$ in rats and in human beings have been reported elsewhere. ${ }^{10}$ Comparison of peptides at which the two monoclonal antibodies are directed showed that the peptide that corresponds to MAb $11 \mathrm{E} 12$ is identical in rats and in humans beings, ${ }^{6}$ whereas the peptide that corresponds to MAb 8E1 differs from one to the other by only one amino acid. As mentioned earlier, the colored reaction occurs only if both monoclonal antibodies are bound to the protein. Second, we performed a preliminary study, in the same manner as explained in the Methods section, to show whether this assay was able to detect cardiac troponin I in the coronary effluent from isolated rat hearts during reperfusion and to study cardiac troponin I release in acute myocardial infarction. In the control group $(n=6)$, the hearts were suspended immediately on the perfusion apparatus. In the ligation group $(n=6)$, the hearts were suspended on the perfusion apparatus immediately after ligation of the first diagonal artery. Results showed that cardiac troponin I was detectable in the coronary effluent from isolated rat hearts and that its concentration was significantly higher in the ligation group than in the control group $(0.40 \pm$ $0.25 \mu \mathrm{g} / \mathrm{L}$ versus $0.06 \pm 0.05 \mu \mathrm{g} / \mathrm{L}$, respectively; $p<$ 0.05 ) in all samples taken within 2 hours of reperfusion. Although the analogy of sequences and the preliminary study do not present a certitude, they do lead to a strong presumption that this assay is valid in rats.

Cardiac troponin I release and myocardial infarction. Cardiac troponin I has been shown to be a reliable marker in myocardial infarction. ${ }^{1-4}$ This marker is more specific than CK-MB, LD, and myoglobin. ${ }^{11}$ In cardiac surgery, cardiac troponin I is efficient in the diagnosis of perioperative myocardial infarction. Mair and associates ${ }^{12}$ showed that cardiac troponin I increased in all patients after coronary artery bypass grafting, but the concentrations were higher $(>3.0 \mu \mathrm{g} / \mathrm{L}$ at 12 hours $)$ in patients with perioperative myocardial infarction. Serum levels of cardiac troponin I were lower in perioperative nonQ-wave myocardial infarction than in perioperative Q-wave myocardial infarction. ${ }^{12}$

Cardiac troponin I and myocardial injury. The fact that cardiac troponin I increased in all patients after coronary bypass reflected the inevitable myocardial damage caused by cardioplegic arrest, ${ }^{12}$ which tends to demonstrate that cardiac troponin I could also be a marker of myocardial ischemia. In a previous study, we ${ }^{5}$ compared postoperative cardiac troponin I concentrations in patients undergoing aortic valve replacement with normal coronary arteries and the concentrations in patients undergoing coronary bypass. Results showed the cardiac troponin I concentration to be significantly higher at hour 6 in the coronary bypass group than in the valve replacement group. Moreover, in the valve replacement group there was a linear correlation between cardiac troponin I concentration at hour 6 and aortic crossclamp time. Although statistically significant $(p<0.01)$, this correlation was modest $(r=$ $0.6)$. No such relationship existed in patients undergoing coronary bypass because, as opposed to aortic valve replacement, wherein myocardial ischemia is only due to cardioplegic arrest, in bypass grafting its cause is multifactorial. Mair and colleagues, ${ }^{12}$ in the study concerning patients undergoing coronary artery bypass grafting, concluded that a wide range of myocardial damage, even in nonperioperative myocardial infarction, is common and not always indicated by CK-MB mass or activity. Cardiac troponin I measurements can detect these small differences in myocardial tissue damage.

Adams and coworkers ${ }^{13}$ showed a good percentage of concordance between cardiac troponin I and CK-MB release after acute myocardial infarction. In his study, five patients without myocardial infarction and no increase of CK-MB had increased cardiac troponin I concentration. Each of these five patients had had episodes of angina before the symptoms that led to their hospitalization. The increase of cardiac troponin I in these patients may have been due to myocardial injury related to unstable angina.

The aim of this study was to show whether there was a link between the duration of ischemia and the release of cardiac troponin I in an experimental 
model of isolated rat hearts. A histologic examination was performed to evaluate myocardial injury.

Cell repartition of cardiac troponin I and release kinetics. Cardiac troponin I circulating levels are normally low, but they rise relatively rapidly after acute myocardial infarction, suggesting the presence of a more accessible pool, perhaps troponin synthesized in the cytosol. ${ }^{14}$ For Haider and Stimson, ${ }^{15}$ the relatively early release of cardiac troponin I could be the result of rapid myofibrillar breakdown, but it may also reflect the presence of free cardiac troponin I within the sarcoplasm that has not yet been incorporated into the myofibrillar texture. Adams and associates ${ }^{13}$ studied the distribution of cardiac troponin I in human heart tissue. The cytosol fraction was $3 \%$ and the myofibril fraction was $97 \%$. Similar percentages of cardiac troponin I were measured in the fractions of cows and dogs. Cardiac troponin I shows a prolonged biphasic release pattern, which is probably the result of the loss of the cytoplasmic pool in the first instance and possibly derived from myofibrillar degradation in the latter.

In our study, the concentration of cardiac troponin I was significantly higher in group III than in groups I and II and in group II compared with group I.

The concentration of cardiac troponin I increased as the ischemic period increased. CK-MB and LD concentrations did not show a similar evolution. They did not increase significantly as the ischemic period increased; in particular, CK-MB concentration was similar after 3 hours and after 6 hours of ischemia. Cardiac troponin I concentration was nearly identical in all samples taken during reperfusion, whereas CK-MB and LD concentrations decreased rapidly. These observations tend to demonstrate either that CK-MB and LD are not reliable markers of myocardial ischemia or that their release is less rapid, mainly because of their higher molecular weight (CK-MB 80,000 kd, LD 180,000 kd, and cardiac troponin I $28,000 \mathrm{kd}) .{ }^{16}$ Indeed, in buffer systems such as the Langendorff preparation, early measurements reflect the cytosolic release of proteins, and proteins with a lower molecular weight are released faster. Sampling late after reperfusion would have made it possible to prove whether or not CK-MB and LD are indeed markers of myocardial ischemia. Other studies, sampling later, showed that $\mathrm{CK}$ and LD concentrations peaked 5 minutes after reperfusion and decreased rapidly thereafter. ${ }^{17,18}$

Release kinetics of CK and LD in the Langendorff preparation of isolated rat hearts. Yamahara and colleagues ${ }^{17}$ designed a study in which ischemic duration was 20 and 60 minutes at $37^{\circ} \mathrm{C}$. The release kinetics of $C K$ and $L D$ showed a monophasic pattern. The peaks in CK and LD release occurred just 5 minutes after stabilization of the hearts, and the levels after 4 hours of reperfusion returned to baseline levels. In the study by Remppis and coworkers ${ }^{18}$ the hearts were damaged either by the calcium paradox or by 60 minutes of no-flow ischemia. Peak levels occurred 5 minutes after the onset of reperfusion with calcium-containing buffers and returned to $1.3 \%(\mathrm{CK})$ and $1 \%(\mathrm{LD})$ of their respective peak levels within 55 minutes of reperfusion. During no-flow ischemia, $\mathrm{CK}$ and $\mathrm{LD}$ rapidly declined to $18 \%(\mathrm{CK})$ and $23 \%$ (LD) of their respective 5-minute values at the end of reperfusion. The release kinetics of CK-MB and LD are similar in our study.

Fig. 1 shows that CK-MB and LD concentrations were maximal at the end of stabilization and decreased thereafter until the sample taken at 90 minutes. The values of CK-MB at 120 minutes seem to be nonrelevant inasmuch as the dispersion of the values was important, as shown by the large confidence interval.

Myocardial injury and infarction. As stated earlier, our measurements probably reflected only the cytosolic release of cardiac troponin I and not the troponins that are bound to the contractile apparatus. Therefore we cannot differentiate injury from infarction. The histologic study showed that the increase of cardiac troponin I might effectively correspond to a myocardial injury. When cardiac troponin I concentrations in hearts in which more than $10 \%$ of myocytes were affected (group B) were compared with hearts in which fewer than $10 \%$ of myocytes were affected (group A), the concentration of cardiac troponin I was clearly higher in group B. Even if the difference was not statistically significant because of the small number of hearts included in group B, there was a tendency to confirm the sensitivity of cardiac troponin I to myocardial injury. Inasmuch as the histologic study was performed too early, it could not discriminate between cells that are viable, but ischemically injured, as opposed to necrotic. Results showed similar and substantial LVPs among the three groups despite the long duration of ischemia (6 hours) for some of them. The statistically significant difference in heart rate between group II and group III (330 \pm 16 versus $280 \pm 11$ beats/min, respectively) is of no clinical significance. 


\section{Conclusion}

This experimental study showed that (1) cardiac troponin I is an early marker of ischemic injury and (2) cardiac troponin I concentration increases as the ischemic period increases. Statistical analysis revealed that the relation between ischemic duration and cardiac troponin I release tended to be linear. Early cardiac troponin I release appears to correlate with the extent of ischemic injury in rats undergoing buffer perfusion. Further evaluation of the release of these cardiac markers in other animal preparations and in human beings could be useful to assess myocardial protection during cardiac operations.

\section{REFERENCES}

1. Larue C, Calzolari C, Bertinchant JP, Leclercq F, Grolleau R, Pau B. Cardiac-specific immunoenzymometric assay of troponin $\mathrm{I}$ in the early phase of acute myocardial infarction. Clin Chem 1993;39(6):972-9.

2. Mair J, Wieser Ch, Seibt I, et al. Troponin T to diagnose myocardial infarction in bypass surgery. Lancet 1991;337: 434-5.

3. Mair J, Wagner I, Puschendorf B, et al. Cardiac troponin I to diagnose myocardial injury. Lancet 1993;341:838-9.

4. Adams JE, Bodor GS, Davila-Roman VG, et al. Cardiac troponin I: a marker with high specificity for cardiac injury. Circulation 1993;88:101-6.

5. Etievent JP, Chocron S, Toubin G, et al. The use of cardiac troponin I as a marker of perioperative myocardial ischemia. Ann Thorac Surg 1995;59:1192-4.

6. Larue C, Defacque-Lacquement $\mathrm{H}$, Calzolari C, Le Nguyen D, Pau B. New monoclonal antibodies as probes for human cardiac troponin I: epitopic analysis with synthetic peptides. Mol Immunol 1992;29:271-8.
7. Rosalki SB. An improved procedure for serum creatine phosphokinase. J Lab Clin Med 1967;69:696-700.

8. Wroblewski F, LaDue JS. Lactic dehydrogenase in blood. Proc Soc Exp Biol Med 1955;90:210-3.

9. Armitage P, Berry G. Rank correlation. In: Statistical methods in medical research. 2nd ed. Oxford: Blackwell Scientific Publications, 1987:417.

10. Wu Q, Raychowdhury MK, Jha PK, Leavis PC, Sarkar S. Characterization of a rabbit fast skeletal troponin I cDNA: a comparative sequence analysis of vertebrate isoforms and tissue-specific expression of a single copy gene. J DNA Seq 1993;4:113-21.

11. Larue C, Calzolari C, Lagrange MC, Pau B. Immunoenzymometric assay of human cardiac troponin I. Immunoanal Biol Spec 1993;8:168-76.

12. Mair J, Larue C, Mair P, Balogh D, Calzolari C, Puschendorf B. Use of cardiac troponin I to diagnose perioperative myocardial infarction in coronary artery bypass grafting. Clin Chem 1994;40:2066-70.

13. Adams JE, Schechtman KB, Landt Y, Ladenson JH, Jaffe AS. Comparable detection of acute myocardial infarction by creatine kinase $\mathrm{MB}$ isoenzyme and cardiac troponin I. Clin Chem 1994;40:1291-5.

14. Adams JE, Abendschein DR, Jaffe AS. Biochemical markers of myocardial injury. Is MB creatine kinase the choice for the 1990s? Circulation 1993;88:750-63.

15. Haider KH, Stimson WH. Cardiac troponin I: a biochemical marker for cardiac cell necrosis. Dis Markers 1993;11:205-15.

16. Hornykewycz S, Gabriel H, Huber K. Biochemical markers of myocardial necrosis in acute myocardial infarction and thrombolysis. Ann Hematol 1994;69(Suppl II):59-63.

17. Yamahara Y, Asayama J, Ohta B, et al. Release kinetics and correlation with hemodynamic dysfunction of cardiac troponin $\mathrm{T}$ in coronary effluent from isolated rat hearts during reperfusion. Basic Res Cardiol 1993;88:307-13.

18. Remppis A, Scheffold T, Greten J, et al. Intracellular compartmentation of troponin $\mathrm{T}$ : release kinetics after global ischemia and calcium paradox in the isolated perfused rat heart. J Mol Cell Cardiol 1995;27:793-803. 